\title{
¿Por qué la interacción? Una reconstrucción de los escritos tempranos de Erving Goffman
}

\author{
Why interaction? A Reconstruction of Erving Goffman's Early Works
}

DOI 10.15517/rr.v99i1.35308

Juan Pablo Gonnet ${ }^{1}$

${ }^{1}$ CIECS / CONICET / Universidad Nacional de Córdoba, Argentina, juanpablogonnet@gmail.com

Fecha de recepción: 23 de noviembre de 2018

Fecha de aceptación: 20 de junio de 2019

\section{Resumen}

El propósito de este artículo es el de reconstruir el camino que llevó a Erving Goffman a la delimitación de la interacción como un campo problemático para la sociología y de aquí, al reconocimiento de un tipo de ordenamiento social que le sería constitutivo. Para esto desarrollamos un análisis en profundidad de los escritos previos a La presentación de la persona en la vida cotidiana. Desde nuestra perspectiva, la teoría de la interacción presentada en este primer texto del autor se configura como una respuesta a un conjunto de problemas que se van a ir definiendo en investigaciones anteriores. El objetivo de este trabajo es sistematizar y organizar estas aproximaciones preliminares. A partir de esta reconstrucción se hará observable que el análisis de la interacción no constituye un objetivo en sí mismo para el proyecto goffmaniano, sino que representa un aporte clave para una teoría general de la sociedad y del orden social.

Palabras clave: Consenso, Individuo, Orden Social, Ritual, Teoría sociológica.

\begin{abstract}
The purpose of this article is to reconstruct the path that led Erving Goffman to the delimitation of interaction as a problematic field to sociology and from this, to the acknowledgement of a social order that would be constitutive to it. In this way, we analyze the essays and articles that are previous to The presentation of self in the everyday life. From our perspective, the theory of interaction presented in this first author's book appears as a conceptual answer to a set of problems that were defined in previous researches. The goal of this paper is to organize and systematize these preliminary approximations. Throughout this reconstruction it will be noticeable that the exploration of interaction does not constitute an end in itself for the goffmanian project, but it represents a central contribution to a general theory of society and of social order.
\end{abstract}

\section{(ब)(1) $(9$}

Este obra está bajo una licencia de Creative Commons Reconocimiento-NoComercial-SinObraDerivada 4.0 Internacional. 
Key words: Consensus, Individual, Social Order, Ritual, Self, Social theory.

\section{Introducción}

La interacción social, entendida como el conjunto de influencias recíprocas que se desencadenan en toda situación en la que dos o más personas se encuentran en presencia física inmediata, ha constituido, indiscutiblemente, el objeto de investigación privilegiado de la obra de Erving Goffman. El sociólogo de origen canadiense analizó en profundidad a este tipo de relación social, así como sus principales características, reglas, normas, mecanismos, lógicas de funcionamiento y el tipo de acción social que le es constitutivo. A pesar de que estos aportes han sido ampliamente reconocidos, frecuentemente han sido disociados del problema de investigación que los inspiró y al que buscaron dar respuesta. En la recepción de los trabajos de Goffman se suele observar un cierto encantamiento por la profundidad y la perspicacia de sus descripciones y conceptualizaciones (Alexander 2000; Boltanski 1973; Bourdieu 1983); pero disociadas de la perspectiva teórica en la que se inscriben y a la que pretenden fundamentar. ${ }^{1}$ En contra de estas lecturas, intentaremos mostrar que el problema central que guía los intereses investigativos de Goffman es el de las condiciones de posibilidad del orden social, el cual debía ser revisitado a la luz del reconocimiento de la complejidad involucrada en las dinámicas interactivas.

Nuestro autor encuentra que las teorías del orden social disponibles en la sociología de mitad del siglo XX resultaban inadecuadas para explicar el orden en contextos interactivos y, por tanto, eran insatisfactorias como teorías generales. En el marco de esta constatación, se configura paulatinamente la hipótesis del «orden de la interacción» entendido como un orden de regulación social particular e irreductible a este tipo de situaciones. El propósito de este artículo es reconstruir el modo en que se va desarrollando este problema del orden social en la interacción, y la manera en la que el autor va articulando teóricamente su solución.

Ciertamente, la teoría de Goffman no pretende ser una teoría general o una teoría de la sociedad. En este sentido, el sociólogo canadiense advirtió acerca del error de buscar en la interacción, la respuesta al problema general del orden social, así como también el de reducir la sociedad a una suma o agregado de interacciones. Sin embargo, es claro que una teoría general del orden social estaría incompleta si no considerase a las interacciones como un tipo de ordenamiento específico; del mismo modo que sería parcial una teoría de la sociedad que no tuviera en cuenta las complejas dinámicas interactivas y cómo estas repercuten en su funcionamiento.

\footnotetext{
${ }^{1}$ Sobre este punto han alertado ciertas recepciones (Collins 1980; Giddens 1987; Rawls 1987). También es posible que Goffman no haya hecho demasiado para evitar este devenir de sus planteos al no existir entre sus producciones evidencias de un desarrollo teórico acumulativo, sistemático y coherente (Caballero 1998; Psathas 1980; Smith 2006). Como menciona Treviño (2003, 33-34): « Regrettably, there is no work in which Goffman consolidates his main ideas in a fully developed manner». [Lamentablemente, no hay un trabajo en el que Goffman condense sus principales ideas en una forma completamente desarrollada]. La traducción es propia como todas las siguientes.
} 
Es por esto que resulta poco adecuado recuperar a Goffman sin problematizar ciertos supuestos teóricos más generales. Por esta razón, entendemos que es pertinente remontarse a los orígenes de la teoría del orden de la interacción, ahora con el objetivo de visualizar los problemas teóricos y empíricos que fueron dando lugar a su formulación. Atendiendo a estos momentos preliminares de la teorización, buscamos contribuir a que la propuesta goffmaniana pueda ser aprehendida, interpretada y discutida a partir de coordenadas sociológicas más amplias.

Para el cumplimiento de este objetivo, hemos considerado metodológicamente adecuado remitirnos a los escritos tempranos de Goffman, especialmente a aquellos trabajos escritos con anterioridad a La presentación de la persona en la vida cotidiana, debido a que los contenidos esbozados en esta última obra ya presuponen las conceptualizaciones en torno al orden de la interacción. La teoría dramatúrgica de la acción esbozada en este libro es un punto de llegada de un conjunto de indagaciones previas y preliminares.

Concretamente, seleccionamos tres ensayos que son altamente significativos para nuestro propósito. Así, tomamos el artículo Symbols of class status (1951), un capítulo de su tesis doctoral Orden social e interacción (1991) y el artículo On face-work (1967). Aunque abordamos otros textos producidos por Goffman en este período, entendemos que estos resultan claves para comprender el modo en que se va conformando la tesis del orden de la interacción, y aquellos problemas conceptuales que pretende resolver. Dividimos el artículo en tres apartados que se corresponden con el análisis y la reconstrucción de cada uno de estos trabajos. Veremos que existe una continuidad reflexiva y acumulativa entre los desarrollos que en ellos se presentan.

\section{Estructuras sociales e interacción}

En el artículo Symbols of class status Goffman (1951) sostiene que toda sociedad involucra algún tipo de diferenciación estructural entre clases, posiciones y roles a los que se les atribuyen diversos niveles de prestigio o status. Goffman plantea sin demasiadas justificaciones el hecho de que la actividad cooperativa, basada en la integración entre los distintos status, es un hecho «universal» y característico de la vida social. Es decir, en toda sociedad tiende a presentarse una aparente armonía y coordinación entre los distintos grupos de status. Ahora bien, la pregunta que le interesa atender a Goffman es la de cómo surge este orden en los intercambios diarios, es decir, cómo podemos saber que aquellos con los que interactuamos poseen el status que dicen o aparentan tener y, por lo tanto, resultan merecedores de determinados tratos. A su vez, cómo podemos lograr que los demás reconozcan nuestro status y actúen en consecuencia. Goffman sostiene que, para que pueda darse algún nivel de acuerdo o armonización entre los comportamientos, resulta imprescindible que el status que un individuo se atribuye sea convergente con aquel que le es atribuido por los demás. A esta convergencia, Goffman la denomina «consenso de trabajo»

\section{(ब) (1) $\otimes \Theta$}

Este obra está bajo una licencia de Creative Commons Reconocimiento-NoComercial-SinObraDerivada 4.0 Internacional. 
(working consensus) (Goffman 1951, 294). ${ }^{2}$ Para que este consenso sea viable, resulta crucial una adecuada comunicación acerca del status. ${ }^{3}$ Esta es la función de los "símbolos de status".

Los «símbolos de status» son medios especializados en la comunicación del status. Siendo esperable que las personas que comparten una misma posición tiendan a tener patrones de comportamiento similares (por ejemplo, determinadas formas de vestir, hablar, consumir, opinar, moverse, etc.), estos podrían convertirse, fácilmente, en signos identificadores de esa posición. De esta manera, el uso de símbolos facilita la atribución de status (test de status) en situaciones sociales concretas, facilitando la solidaridad (entre los miembros de un mismo grupo de status o de grupos diferentes). Adicionalmente, los símbolos de status contribuyen a la coordinación en tanto que no sólo permiten situar a una persona en una determinada posición, sino que también brindan información acerca del estilo de vida, los valores culturales y los puntos de vista que se hayan asociados a esa posición. En este sentido, los símbolos no solo tienen un valor categorial, sino también expresivo.

Más allá de esta capacidad comunicativa de los símbolos de status, Goffman no considera que sean suficientes para resolver el problema de la comunicación del status. De hecho, plantea: «This paper is concerned with the pressures that play upon behavior as a result of the fact that a symbol of status is not always a very good test of status» (Goffman 1951, 295). ${ }^{4}$ El problema aparece por el hecho de que los símbolos representan algo que no es directamente asequible en la interacción. Así, nada impediría que fuesen usados de modos fraudulentos. Dados los beneficios que se encuentran aparejados a la posesión de un status determinado, sería factible que un individuo dispusiera de los símbolos de status para aparentar una posición o un rol que no posee en realidad. Esta autonomía es lo que lleva a que ocasionalmente estos no sean buenos indicadores del status. Goffman (1951, 295-296) menciona:

Status symbols are used because they are better suited to the requirements of communication than are the rights and duties which they signify. This very fact, however, makes it necessary for status symbols to be distinct and separate from that which they signify. It is always possible, therefore, that symbols may come to be employed in a fraudulent way, to signify status which the claimant does not in fact possess. We may say, then, that continuing use of status symbols in social situations requires mechanisms for restricting the opportunities that arise for misrepresentation.

\footnotetext{
${ }^{2}$ Este es un concepto que deberemos retener, porque resulta clave en la estructuración de la respuesta al problema del orden social en la interacción.

${ }^{3}$ Vale aclarar que este análisis tiene sentido en una sociedad de clases en las que los símbolos de status no se encuentran garantizados hereditariamente. Por otra parte, también se encuentra el supuesto de que, en la sociedad moderna, resulta poco apropiado la comunicación explícita acerca de nuestro status o posición social en los encuentros sociales (Cadenas 2012; Luhmann 1987).

${ }^{4}$ [Este artículo trata sobre las presiones que se presentan sobre el comportamiento como resultado del hecho de que los símbolos de status no son siempre buenos indicadores del status]
} 
We may approach to the study of status symbols by classifying the restrictive mechanisms embodied in them. ${ }^{5}$

Para Goffman, existen dos tipos de símbolos de status: los ocupacionales y los de clase. Los primeros son menos susceptibles a ser tergiversados, ya que su utilización se encuentra protegida por sanciones legales y resultan más susceptibles de ser corroborados por informaciones disponibles (por ejemplo, el currículum, títulos universitarios, posiciones formales en una organización, etc.). Los símbolos de clase, en cambio, son menos controlables institucionalmente al no encontrarse referidos a una única fuente de status sino a un conjunto de ellas. De hecho, cuando un individuo manipula un símbolo de status de clase de un modo fraudulento, no podemos probar tan fácilmente que este sea el caso.

Sumado a esto, nuestro juicio acerca de las posiciones de clase de otros es complejo, ya que involucra la necesidad de sopesar distintas fuentes de información, lo que siempre da lugar a un margen de duda. Por otra parte, los derechos y beneficios percibidos por la posesión de un status de clase no se hayan oficialmente aprobados por leyes o contratos, por lo que los desviados no cometen un delito sino un «atrevimiento». De aquí que Goffman llegue a decir que la función de estos símbolos es más la de influenciar el juicio de los otros en una dirección deseada, que la de representar una posición de clase determinada.

Para evitar los riesgos de tergiversación que habilitan los símbolos de clase, Goffman observa que su uso suele estar condicionado por un conjunto de restricciones («restrictive mechanisms»). Una primera restricción se encuentra en el empleo de aquellos símbolos intrínsecamente conectados con su uso. Por ejemplo, símbolos asociados a la posesión de cierto nivel de poder o de ingresos. Es difícil sostener estas representaciones si las prácticas no lo corroboran.

También existen limitaciones ligadas a la socialización. En este sentido, todos aquellos ítems de comportamiento que son naturales para los miembros de una clase, como las formas de moverse, hablar, vestir o gesticular, son difíciles de ser imitadas por aquellos que no han sido socializados en ella. Por último, podemos mencionar aquellas restricciones que remiten a la escasez natural de determinados objetos simbólicos, tanto materiales (obras de arte, joyas y otros materiales relativamente escasos) como inmateriales (pertenencia a cierta familia, posesión de determinadas amistades o contactos, o la participación en determinados eventos considerados únicos o

\footnotetext{
${ }^{5}$ [Los símbolos de status son usados porque se encuentran mejor preparados para los requerimientos de comunicación que los derechos y las obligaciones que ellos representan. Este hecho, sin embargo, hace necesario que los símbolos de status sean algo distinto y separado de aquello que significan. Siempre es posible, así, que los símbolos puedan ser empleados de un modo fraudulento, esto es, representando un status que el demandante no posee. Podríamos decir, que el uso continuado de símbolos de status en situaciones sociales requeriría mecanismos que restrinjan las oportunidades de mal-representación. Podríamos aproximarnos al estudio de los símbolos de status clasificando los mecanismos restrictivos que se encuentran incorporados en ellos.]
} 
irrepetibles, entre otras). ${ }^{6}$ En conexión con esto, es de esperar que los ítems seleccionados para funcionar como símbolos de status de clase involucren al menos alguno de estos mecanismos restrictivos. A pesar de esto, las restricciones siempre pueden ser sorteadas; es decir, no pueden asegurar que el símbolo de estatus utilizado por un individuo sea un indicador adecuado del status de clase. $^{7}$

Consideramos que en esta indagación se van a perfilar dos avances importantes para el desarrollo de la futura teoría del orden de la interacción. Por un lado, se reconoce a la interacción social (incluso sin llamarla así) como un campo problemático específico e irreductible a los problemas generales de organización de la sociedad. De hecho, Goffman pareciera adherir a dos teorías de la sociedad, la de la división del trabajo y la de la estratificación, las cuales considera complementarias y válidas para el abordaje del funcionamiento general de la sociedad, pero que se mostrarían insuficientes para la comprensión de la interacción cotidiana. En esta dirección, los símbolos de status de clase no se presentan como un mero correlato de las posiciones de clase, sino que aparecen como medios de comunicación necesarios para la coordinación interactiva. En gran parte de nuestros encuentros sociales carecemos de información suficiente acerca del status de los otros; del mismo modo que ellos carecen de información acerca del nuestro. De este modo, precisamos de los símbolos de status como medios para guiar nuestras acciones y las de los otros. Estos constituyen el único acceso que disponemos a la posición social del otro. No obstante, al ser representaciones del status de clase y no su mero reflejo, el uso de símbolos de status debe ser regulado por mecanismos adicionales.

Por otro lado, un segundo aporte del artículo en dirección a una teoría del orden de la interacción, se encuentra en la demostración de una cierta autonomía de la interacción con respecto a las estructuras sociales. Frecuentemente, se ha interpretado a este análisis goffmaniano como un modo de resaltar la capacidad individual de los actores por tergiversar, burlar y manipular estratégicamente sus posicionamientos sociales (Winkin 1991). No obstante, consideramos que esta lectura no resulta del todo adecuada. Podríamos decir, por el contrario, que Goffman busca mostrar lo dificultoso, laborioso y arriesgado que puede resultar para un individuo cualquier intento por tergiversar su posición social. ${ }^{8} \mathrm{El}$ análisis de los mecanismos restrictivos es revelador en este

\footnotetext{
${ }^{6}$ Además de estos, Goffman determina otros tres mecanismos: las restricciones morales (asociadas al sentido del lugar que ocupa un individuo), las restricciones temporales (asociadas al tiempo de dedicación, atención y disciplina requerida para la posesión de un status) y, finalmente, las restricciones orgánicas (ligadas a las marcas físicas o corporales asociadas a una posición).

${ }^{7}$ Goffman destaca el papel de ciertas profesiones que contribuyen al logro de este desafío. Por ejemplo, los diseñadores de moda, los arquitectos, los decoradores de interiores y otro tipo de asesores. Incluso las restricciones mismas, pueden llevarnos al error en contextos de movilidad social ascendente o descendente.

${ }^{8}$ «As a result of the circulation of symbols, however, a sign which is expressive for the class in which it originates comes to be employed by a different class - a class for which the symbol can signify status but ill express it. In this way conscious life may become thin and meager, focused as it is upon symbols which are not particularly congenial to it» (Goffman 1951, 304). [Como resultado de la circulación de símbolos, un signo que es expresivo para la clase en que se origina es empleado por una clase diferente - una clase para la que el símbolo puede significar status pero
}

\section{(C) $(1) \Theta$}

Este obra está bajo una licencia de Creative Commons Reconocimiento-NoComercial-SinObraDerivada 4.0 Internacional. 
punto al exhibir el inmenso trabajo social por controlar el fraude en el manejo de los símbolos de status. ${ }^{9}$

Lo que realmente le parece significativo a Goffman no es tanto la tergiversación en sí, sino aquella condición que habilita esta alternativa. La posibilidad de aparentar un status que no se posee, no se debe a destrezas o suspicacias individuales, sino que es factible en tanto la interacción se encuentra parcialmente autonomizada de las estructuras sociales de la sociedad. Si no existiera esta autonomía de la situación interactiva, no serían necesarios mecanismos de control específicos; los símbolos serían representables sólo por aquellos que ocupasen determinada posición social (serían indicadores confiables del status). No habría separación entre clase y símbolo de status.

Para ilustrar esto, podemos retomar una situación empírica descripta por Goffman. En contextos de movilidad social ascendente o descendente, los individuos o los grupos sociales se desplazan en la estructura de clases. Sin embargo, gracias a los símbolos y a sus mecanismos restrictivos, estos desplazamientos no impactan inmediatamente en el status de estos grupos. En estas transiciones, los símbolos dejan de simbolizar una posición de clase para pasar a conferirla. Es así como la interacción puede ralentizar la pérdida de status de un grupo que ha descendido socialmente (estos pueden seguir utilizando, sin mayores problemas y al menos por un tiempo, los símbolos de status propios de la posición de clase perdida) y, a la vez, limitar el acceso a aquellos que han mejorado su situación de clase (al no poder sortear las restricciones propias de los símbolos de status). Este ejemplo muestra no sólo los límites que se ponen en juego en la interacción con respecto a la representación directa de las estructuras sociales, sino que también permite observar algunos de los condicionamientos que las interacciones ejercen sobre las estructuras sociales. Resumiendo estos puntos, encontramos que en este análisis de los símbolos de status de clase se evidencian dos descubrimientos teóricos generales. Estos son 1) el carácter problemático de la coordinación en las comunicaciones cotidianas a pesar de la existencia de un orden societal más o menos estabilizado, y 2) la posibilidad de considerar a la interacción como un ámbito social parcialmente autonomizado de las realidades estructurales e institucionales de la sociedad.

\section{Las particularidades del orden de la interacción}

Habiendo reconocido el problema de la autonomía de la interacción en relación a las estructuras de clases, de roles, de posiciones y de status, surge la pregunta acerca del modo en que es posible el orden social en estas situaciones. Es decir, la manera en que es posible la coordinación entre los comportamientos individuales. Ciertamente, los símbolos de status resultan de gran ayuda, no obstante, como pudimos ver, tienen sus limitaciones. En su trabajo de tesis doctoral titulado

deficientemente expresado. En este sentido, la vida consciente se hace delgada y pobre, focalizada en símbolos que no son particularmente agradables para ella.]

${ }^{9}$ Otro ejemplo es la permanente búsqueda, por parte de las clases o grupos privilegiados, de símbolos de status que no se encuentren contaminados por la apropiación de otras clases.

\section{(c) (1) $\$(9$}

Este obra está bajo una licencia de Creative Commons Reconocimiento-NoComercial-SinObraDerivada 4.0 Internacional. 
Communication in an island community ${ }^{10}$, Goffman sostiene que el orden de la interacción se viabiliza a través de un compromiso circunstancial entre los individuos, que permite superar la multiplicidad de contingencias que, como describimos en el apartado anterior, son constitutivas de las dinámicas interactivas.

Lamentablemente la tesis doctoral de Goffman (1991) no se encuentra publicada. Sin embargo, uno de los capítulos que reviste especial atención para nuestros propósitos si lo está. El capítulo se titula «El orden social y la interacción», título cercano al de la conferencia que iba a impartir ${ }^{11}$ al finalizar su carrera como presidente de la Asociación Americana de Sociología («El orden de la interacción » - Goffman, 1983); lo que demuestra no sólo la recurrencia o insistencia en la temática, sino también el carácter medular de la misma en su proyecto teórico e intelectual. En esta exploración iniciática, Goffman se aproxima a la interacción destacando las limitaciones que presenta el modelo normativo de orden social propuesto por Parsons (1999), hegemónico en la sociología de ese entonces, para explicar la lógica de los encuentros sociales presenciales. Cabría decir que no hay en este trabajo de Goffman una defensa de la tesis del orden de la interacción, sino una exhibición de las deficiencias que tiene la teoría del orden social prevaleciente para el abordaje de la interacción.

Podríamos reconstruir este escrito de Goffman a partir de la consideración de dos aportes que muestran una visible continuidad con los problemas ya esbozados en «Symbols». Por un lado, Goffman caracteriza más detalladamente las interacciones como sistemas distinguibles de la vida en sociedad, algo que no se encontraba del todo presente hasta este momento. Así, se determinan el tipo de acciones, expectativas y límites que les son propios a las interacciones. De esta manera, resulta justificada la posibilidad de diferenciar sus dinámicas con respecto a las propias de otros sistemas sociales. Por otro lado, en un segundo momento, Goffman aborda el problema del orden social en las interacciones, atendiendo a la frecuencia con la que en estas se presentan desviaciones y desacuerdos. La cuestión es cómo es posible el orden social en una situación en la que este parece encontrarse permanentemente amenazado.

Las interacciones se encuentran compuestas por actos de comunicación o mensajes temporalmente encadenados. De aquí que Goffman considere adecuado analizar las interacciones como procesos o dinámicas conversacionales. Los individuos participan en las interacciones debido a que, a través de ellas, pueden cumplir con ciertos fines y objetivos más o menos conscientes. La sociedad, por su parte, requiere que los individuos interactúen en tanto de esto depende, en parte, su reproducción. Lo que garantiza el desarrollo de las interacciones es la expectativa de que los demás contribuirán a su adecuado y normal desarrollo.

\footnotetext{
${ }^{10}$ Esta tesis consistió en una investigación acerca de la interacción entre los habitantes locales y los turistas en las Islas Shetland de Escocia. Los resultados de este trabajo habrían sido centrales para la configuración de su campo de estudio en torno a la interacción (Treviño 2003, 4).

${ }^{11}$ Debido a su fallecimiento, la conferencia no fue pronunciada.
}

\section{(C) $(1) \Theta$}

Este obra está bajo una licencia de Creative Commons Reconocimiento-NoComercial-SinObraDerivada 4.0 Internacional. 
Debe resaltarse que no es una expectativa en torno al comportamiento del otro, sino una expectativa en torno a la contribución al funcionamiento de la interacción. Sin esta expectativa, sería improbable la interacción. No obstante, esta expectativa no es un a priori sino que es una condición puesta permanentemente a prueba y evaluada en la misma situación social. No es una confianza ciega en el otro.

En tercer lugar, Goffman considera que esta expectativa trata de garantizarse a través de sanciones positivas y negativas; pero a diferencia de otros órdenes sociales, estas suelen ser más sutiles y moderadas. Caso contrario podrían interrumpir el flujo conversacional, destruyendo aquello que buscan mantener:

La relación entre el orden conversacional y las sanciones que lo regulan parece algo diferente de la que se establece entre otros tipos de órdenes sociales y sus sanciones reguladoras. En efecto, las sanciones empleadas para mantener el orden conversacional corresponden en gran medida al orden de la aprobación o desaprobación directamente expresada o sentida. No parece que se insista en sanciones de género más instrumental. Por otra parte, y mucho más que en el caso de otros órdenes sociales, el problema del orden conversacional es emplear una sanción cuya simple aplicación no elimine el orden que dicha sanción debe mantener. (Goffman 1991, 93)

Por último, toda interacción social se desarrolla en contextos sociales más amplios (organizaciones, sistemas societales, interacciones sociales mayores, movimientos sociales, etc.), con los cuales mantiene ciertos vínculos de dependencia. A pesar de esto, la interacción tiene sus grados de libertad a partir de ciertos límites que lo separan de esos entornos y le dan autonomía. En este sentido, el funcionamiento de la interacción puede ser más o menos funcional en relación a su medio. Es decir, puede contribuir, condicionar o limitar el funcionamiento de aquellos órdenes sociales al interior de los cuales opera. Esto significa que la sociedad no puede ser concebida ni como una interacción ni como una suma de ellas. La interacción presupone a la sociedad.

Dadas las características de los sistemas interactivos, la pregunta que sigue es cómo se viabiliza el orden social en ellos. Goffman busca la respuesta a este interrogante en el análisis detallado de los procesos que se desencadenan cuando se rompen las reglas de la interacción; es decir, cuando las expectativas en torno al comportamiento interactivo son defraudadas por las acciones de uno de los participantes. Dice Goffman $(1991,94)$ : «Estos actos (...) nos proporcionan la ocasión de estudiar los supuestos en que se basa el comportamiento interaccional adecuado. Estas infracciones del comportamiento correcto nos ofrecen el medio para llevar nuestra atención a las exigencias de las situaciones ordinarias». La descomposición analítica de estas instancias le permite a Goffman vislumbrar aquello que resulta necesario para el funcionamiento ordenado de la interacción.

\section{(c) (1) $\$($}

Este obra está bajo una licencia de Creative Commons Reconocimiento-NoComercial-SinObraDerivada 4.0 Internacional. 
Lo primero que cabe destacar es que, cuando en una interacción conversacional alguien infringe las reglas de conducta, la desorganización o el desorden social se experimenta como embarazo y trae aparejado diversos grados de confusión y desorientación para los participantes. Estos se sienten incómodos, molestos, turbados y la situación social deviene «anómica», en tanto se pierden parámetros para el comportamiento. Más allá de los perjuicios ocasionados por el infractor de las reglas, no se considera a este como un desviado. En las conversaciones se lo suele concebir como torpe, inoportuno o fuera de lugar. Así, los actos que provocan embarazo son «meteduras de pata» (Goffman 1991, 94). Cuando alguien infringe las reglas de la interacción en múltiples situaciones, puede ser considerado como un desviado. Luego, el ofensor siente vergüenza, al igual que todos aquellos que se identifiquen con él. Los ofendidos, por su parte, se sienten agredidos y amenazados.

Frente a estas circunstancias, se hace necesario algún tipo de restructuración o restablecimiento del orden; es decir, se requiere de la puesta en marcha de acciones correctivas. En el caso de las conversaciones, puede darse un conjunto de acciones que buscan proteger a la persona ofendida, pero que involucran la destrucción del orden de la interacción. Por ejemplo, uno se puede marchar, abandonar la interacción, agredir o denunciar al ofensor o, simplemente, desconocerlo. Siendo las interacciones escenarios en los cuales la ofensa es un hecho frecuente, el uso de estas medidas radicales resulta contraproducente. Por esta razón, $\operatorname{Goffman}(1991,95)$ sostiene que otras alternativas suelen ser más habituales: «Los participantes responden lo más a menudo con tolerancia e indulgencia a los delitos contra el orden de la interacción. Aunque esta relación acomodaticia sea bastante precaria, permite el mantenimiento de la interacción. Si deben aplicarse acciones de corrección, pueden hacerse con delicadeza, sin romper la interacción misma».

En esta práctica indulgente, Goffman encuentra una de las claves para dilucidar aquello que hace posible las interacciones. Si ante las desviaciones solemos actuar tolerantemente, esto significa que para que la coordinación interactiva funcione basta con mantener una apariencia de acuerdo entre los interlocutores. En otras palabras, exhibir un cierto grado de aceptación circunstancial del comportamiento del otro. En palabras de Goffman (1991, 95): «La conducta de acomodación toma la forma de una aparente aceptación del comportamiento del otro; lo cual origina lo que podríamos llamar como compromiso de conveniencias (working acceptance)».

Este compromiso de conveniencias resulta fundamental para el orden de la interacción. ${ }^{12} \mathrm{El}$ mismo permite mostrar que no son necesarios consensos profundos entre los participantes de la interacción para que esta funcione adecuadamente, basta con ciertos acuerdos superficiales. El único consenso real entre los individuos es el del deseo de mantener un compromiso de conveniencias. En este punto, Goffman reconoce el papel fundamental que desempeña el "tacto", el cual es entendido como el conjunto de tácticas que son empleadas para cuidar preventiva y

\footnotetext{
${ }^{12}$ Una idea similar ya se encontraba presente en Symbols of class status, a través del concepto de «consenso de trabajo» considerado como un requisito básico para la coordinación entre los grupos de status.
}

\section{(c) (1) $\$(9$}

Este obra está bajo una licencia de Creative Commons Reconocimiento-NoComercial-SinObraDerivada 4.0 Internacional. 
correctivamente al compromiso de conveniencias. Ahora bien, estos consensos de trabajo no constituyen ni una finalidad de las interacciones ni un conjunto de medios para la acción. De hecho, Goffman $(1991,97)$ sostiene que «es preferible entender a la interacción, no como una escena de armonía, sino como una ordenación que permite perseguir una guerra fría». De lo que se trata es de mantener la apariencia de acuerdo «un modus vivendi que permite atender las cosas y los asuntos esenciales» (Goffman 1991, 97).

Veamos, entonces, los avances que estas reflexiones proponen en relación a Symbols... Como mencionamos, en este primer trabajo, Goffman deja planteado el problema que representa la interacción para el orden social. En esta dirección, el texto analizado en este apartado profundiza en la especificación de las características de la interacción, la cual pasa a ser concebida más enfáticamente como un sistema o un orden social con sus particularidades (tipo de actos, límites, expectativas y sanciones). Esta clarificación permite atender a la pregunta por el modo en que se organizan estos órdenes sociales.

Las expectativas que los viabilizan resultan permanentemente amenazadas por desviaciones, disrupciones, discrepancias, ofensas y desacuerdos. Las interacciones deben lidiar con estos hechos, pero sin que se vea comprometida su misma existencia. La tesis goffmaniana es que las interacciones resuelven este problema a partir de la mantención de un «compromiso de conveniencias» o «acuerdo de trabajo», que prescinde de la necesidad de consensos profundos. Algo que quedará pendiente es la pregunta por las razones que llevan a sostener y a respetar estos compromisos. Acerca de este punto, Goffman $(1991,97)$ sólo menciona: «En general, podemos confiar en que la gente hará una cosa: hará todo lo posible por evitar una escena». Este argumento resulta, claramente, insuficiente y, por esta razón, será la temática de un artículo inmediatamente posterior.

\section{La dimensión ritual de la interacción}

En su tesis doctoral, Goffman reconoce que el orden de la interacción resulta viable por los acuerdos o consensos de trabajo. Sin embargo, algo que permanece inexplicado es cómo son posibles esos consensos superficiales, aparentes, circunstanciales y pragmáticos. En otros términos, no da respuesta acerca de las razones por las cuales estos acuerdos son aceptados y respetados. ¿Qué nos lleva a comprometernos con ellos? En el ensayo On face work. An analysis of ritual elements in social interaction, el sociólogo canadiense propone una respuesta a esta interrogante. Aquí Goffman postula que existe una dimensión moral que es constitutiva del sistema interaccional y que tiene que ver con en el cuidado de la «cara» o del «social-self» de las personas que en ella

\section{(ब) (1) $(9$}

Este obra está bajo una licencia de Creative Commons Reconocimiento-NoComercial-SinObraDerivada 4.0 Internacional. 
participan. ${ }^{13}$ Para el autor, la cara representa un objeto sagrado que debe ser protegido y resguardado en los encuentros sociales. Goffman llega a esta constatación a partir de la observación del carácter ritual de ciertas prácticas, estrategias y comportamientos interaccionales que exhiben una preocupación especial por la preservación de los interlocutores. ${ }^{14}$ Este descubrimiento será fundamental para explicar las condiciones de posibilidad de los acuerdos de trabajo en los que se basa el orden de la interacción.

En los encuentros sociales presenciales (cara a cara), la primera preocupación de los individuos es, precisamente, salvar o proteger sus caras frente a una posible situación de degradación o descrédito. La aceptación de los acuerdos de trabajo es posible porque el principal constreñimiento en la interacción proviene de las exigencias del cuidado de la cara. Adherimos a estos acuerdos porque, a través de ellos, la cara de los interactuantes se encuentra suficientemente protegida. Los acuerdos son el resultado de este orden moral; Goffman propone que el cuidado de la cara es funcional al orden de la interacción. Abordemos esta hipótesis más de cerca.

En contextos de presencia física inmediata, los individuos expresan una visión de la situación, de los otros y de sí mismos. Esto puede ser realizado de manera consciente o inconsciente y basta con que esta atribución sea hecha por los demás a las expresiones emanadas por alguien. Por lo general, se asume que el otro definió una perspectiva, haya sido esta escogida intencionalmente o no. Goffman considera que las impresiones nos permiten asignarle una cara (o una pretensión de ella) a una persona, la cual es definida como «...the positive social value a person effectively claims for himself by the line others assume he has taken during a particular contact»

\footnotetext{
${ }^{13}$ Es necesario aclarar que, para Goffman, el self no describe a algo que sea propiedad del individuo, sino que es un producto social, es algo que se constituye en el proceso mismo de la interacción y del encuentro social (Treviño 2003, 14-15).

${ }^{14}$ En un artículo anterior, Goffman $(1952,463)$ reconoce esta dimensión en la descripción de las diversas estrategias que se ponen en marcha para consolar a aquellos individuos que, por distintas razones, hubiesen perdido (o estuviesen por perder) su cara o su social-self.

.... a person is an individual who becomes involved in a value of some kind a role, status, a relationship, an ideology and then makes a public claim that he is to be defined and treated as someone who possesses the value or property in question. The limits of his claims, and hence the limits to his self, are primarily determined by the objective facts of his social life and secondarily determined by the degree to which sympathetic interpretation of these facts can bend them in his favor. Any event which demonstrates that someone has made a false claim, defining himself as something which he is not, tends to destroy him. If other realized that the person's conception of self has been contradicted and discredited, the person tends to be destroyed in the eye of others (463). [...una persona es un individuo que se encuentra involucrado en el valor de un determinado rol, status, relación, o ideología y luego es aquel que hace un reclamo de que él debe ser definido y tratado como alguien que posee el valor o la propiedad en cuestión. Los límites de su reclamo, y de aquí los límites de su persona, son primariamente determinados por los hechos objetivos de la vida social y luego por el grado en que la interpretación de estos hechos puede favorecerlo. Cualquier evento que demuestre que alguien ha hecho un reclamo falso, definiéndose a sí mismo como alguien que no es, tiende a destruirlo. Si otros se dan cuenta que la concepción de sí mismo que tiene la persona ha sido contradicha y desacreditada, la persona tiende a ser destruida ante la mirada de los demás].
} 
(Goffman 1967, 5). ${ }^{15}$ Podría decirse que la cara es una imagen socialmente aprobada de uno mismo. A partir de esta atribución, las conductas y comportamientos de los actores pueden observarse como en mayor o menor concordancia con la cara. En tanto que la cara es asignada por los otros, el individuo debe exhibir un compromiso con ella a través de líneas de acción consistentes y coherentes (face work), evitando informaciones disidentes que pongan en duda las expectativas generadas. De este modo, el compromiso con mi cara es una responsabilidad que tengo para con los otros.

En toda situación de interacción deseamos que se nos valore y reconozca de determinada manera, pero esto involucra la obligación a actuar de un modo convincente con esa pretensión (mostrar que uno es lo que dice ser). Los otros sostendrán nuestra cara si hacemos los debidos esfuerzos. Cuando los individuos no son lo suficientemente cuidadosos pueden «perder su cara», sintiéndose avergonzados o fuera de lugar. ${ }^{16}$ De alguna manera, esta condición promueve un cierto grado de autocontrol de los individuos en la interacción.

Ciertamente, podríamos pensar que cualquiera podría reclamar cualquier cara, cuestión que Goffman ya reconocía en su análisis de los símbolos de status. El compromiso moral que las personas tienen con su cara representa un importante límite a esta posibilidad. Tergiversar los hechos puede acarrearnos serios riesgos. Por ejemplo, si los otros descubriesen que nos encontramos sustentado valores, status o roles que no se corresponden con nuestra existencia real en el mundo social, esto podría llevarnos a perder la cara y, así, al descrédito y la desconfianza por parte de nuestros copartícipes en la interacción. ${ }^{17}$ En relación a esto, $\operatorname{Goffman}(1967,10)$ menciona: «while social face can be his most personal possession and the center of his security and pleasure, it is only a loan to him from society; it will be withdrawn unless he conducts himself in a way that is worthy of it. Approved attributes and their relation to face make every man his own jailer; this is a fundamental social constraint even though each man may like his cell». ${ }^{18}$

Así, el componente moral de la interacción tiene que ver con una expectativa acerca del comportamiento honorable, digno y respetuoso en un encuentro social. El no cumplimiento de estas expectativas implica el descuido de la cara y deviene en una desviación con respecto a este orden moral.

A pesar de esto, Goffman considera que no estamos solos en el cuidado de la cara. Por cierto, nuestros esfuerzos por mantener la cara se encuentran apoyados por la consideración y la

\footnotetext{
15 [...el valor positivo que una persona efectivamente define para sí mismo por la línea que otros asumen él ha tomado durante un contacto particular].

${ }^{16}$ Un análisis sistemático de la vergüenza y su función en los encuentros sociales se encuentra en Goffman (1956a).

${ }^{17}$ Notablemente, este problema se hace más agudo cuando se considera la posibilidad de volver a encontrarme con aquellos con los cuales estoy interactuando.

${ }^{18}$ [aunque su cara social pueda ser su posesión más personal y el centro de su seguridad y placer, es solamente un préstamo para el de la sociedad, esta será retirada a menos que él se conduzca de un modo que sea merecedor de la mismo. Atributos aprobados y su relación con la cara hacen de cada hombre su propio carcelero; esto es un constreñimiento social fundamental incluso cuando a cada hombre le guste su celda.]
} 
cortesía de los demás. En tanto que nuestra cara es atribuida por los otros, es de esperar que ellos también se sientan responsables por ella y exista un esfuerzo por preservarla y cuidarla. Esto es no dejar que perdamos la cara. Goffman muestra que aquel que puede ser testigo de la humillación del otro, manteniendo una cierta frialdad, suele ser considerado como alguien descorazonado (alguien que no se moviliza por la cara de los demás). También sería posible pensar que una persona puede perder su cara cuando no se (pre) ocupa de la cara de los demás. Por otra parte, aquel que no tiene problemas en perder su cara es un "sinvergüenza" (descarado). Adicionalmente, debido a que la cara de los demás depende de nosotros, es de esperar que la eventual pérdida de nuestra cara ponga en riesgo la de ellos. De esta manera, el respeto a la cara se manifiesta tanto en el cuidado de la propia como en la ajena. Esta preocupación mutua por la cara del otro es clave para el orden de la interacción, ya que permite que en la mayoría de las ocasiones aceptemos la línea de conducta que ha sido tomada por un participante. Goffman $(1967,22)$ lo expresa de la siguiente manera: «A state where everyone temporarily accepts everyone else's line is established. This kind of mutual acceptance seems to be a basic structural feature of interaction, especially the interaction of faceto-face talk. It is typically a working acceptance, not a real one, since it tends to be based not on agreement of candidly expressed heart felt evaluations, but upon willingness to give temporary lip service to judgments with which the participants do not really agree». ${ }^{19}$

Es así como el mantenimiento y la protección de la cara da lugar a los consensos de trabajo que en artículos previos Goffman había reconocido como fundamentales para el orden de la interacción. Los acuerdos de trabajo resultan de las prácticas de respeto y cuidado de la cara. Por consiguiente, existe un vínculo estrecho entre la protección de la cara y el orden de la interacción. Lo primero es condición de lo segundo. «Since each participant in an undertaking is concerned, albeit for different reasons, with saving his own face and the face of others, then tacit cooperation will naturally arise so that the participants together can attain their shared but differently motivated objectives» (Goffman 1967, 29). ${ }^{20}$

Los acuerdos de trabajo, como instancias de aceptación mutua de comportamientos, devienen en hechos morales. La amenaza a estos acuerdos es una amenaza a la cara y, por lo tanto, una amenaza contra el orden moral en el que se sustentan y al cual buscan proteger. Por lo tanto, una amenaza a la cara deviene en un riesgo para la reproducción de interacción. Atendiendo a esto, Goffman sostiene que las reglas de la interacción identificables en la vida cotidiana describen, fundamentalmente, pautas que prescriben un comportamiento atento a la cara (por ejemplo, las

\footnotetext{
${ }^{19}$ [Un estado en el que todos temporariamente aceptan las líneas de todos es establecido. Esta clase de aceptación mutua parece ser una característica estructural básica de la interacción, especialmente la interacción, especialmente la interacción cara-a-cara. Es típicamente una aceptación práctica, no una real, en tanto tiende a estar basada no en un acuerdo de evaluaciones profundas, sino en la complacencia de brindar una aceptación circunstancial a juicios con los que los participantes no acuerdan realmente.]

${ }^{20}$ [En tanto que cada participante se preocupa, aunque por diferentes razones, con salvar su propia cara y la cara de los demás, la cooperación tácita emergerá naturalmente para que los participantes puedan alcanzar sus objetivos compartidos, pero diferentemente motivados.]
} 
reglas ceremoniales de cortesía y de decoro $\left.{ }^{21}\right)$. En tanto que estas pautas pretenden proteger la sacralidad de la cara, Goffman $(1967,19)$ no duda en concebir a las prácticas que las ejecutan como rituales. «I use the term ritual because I am dealing with acts trough whose symbolic component the actor shows how worthy he is of respect or how worthy he feels other are of it. The imagery of equilibrium is apt here because the length and intensity of the corrective effort is nicely adapted to the persistence and intensity of the threat. One's face, then, is a sacred thing, and the expressive order required to sustain it is therefore a ritual one». ${ }^{22}$ Por ejemplo, el saludar al arribar a un encuentro social puede ser entendido como un ritual en tanto es un acto que exhibe consideración hacia la cara del otro.

Para el mantenimiento de la cara existen diversos tipos de rituales. Por un lado, Goffman destaca aquellas prácticas que buscan prevenir amenazas a la cara. Por ejemplo, las que tienen que ver con el evitar ciertos contactos, temáticas o situaciones que podrían ser incompatibles con mi cara o la de los otros. Esto requiere que los individuos actúen con discreción, tacto y cortesía; incluso cuando se presentan determinadas acciones o comportamientos que ofenden a la cara y que no pudieron ser evitados o prevenidos (un ruido en el estómago en medio de una conversación puede ser pasado por alto, aunque evidentemente implica una amenaza a la cara). En segundo lugar, se encuentran prácticas correctivas, que son utilizadas cuando un incidente no ha podido ser evitado ni pasado por alto. Aquí estamos ante eventos que son difíciles de ser desestimados y, por esta razón, requieren de una atención especial.

$\mathrm{El}$ orden ritual ha sido afectado y es preciso restaurar su equilibrio si se pretende que la interacción continúe. Goffman considera que en estas circunstancias emerge un intercambio (interchange) entre los participantes, en el cual el ofendido llama la atención acerca de la conducta ofensiva y le demanda al ofensor volver a la línea de acción perdida. Es decir, se le ofrece al ofensor la posibilidad de que corrija su conducta y restablezca el orden. En este sentido, el ofensor puede hacer un chiste (quitándole relevancia a su infracción), explicar el error o pedir disculpas; puede buscar compensar a los ofendidos o sancionarse. Sea como fuere, debe demostrar que es una persona renovada y que está dispuesta a pagar por su ofensa. Finalmente, el ofendido puede aceptar la ofrenda y el ofensor puede sentirse aliviado. Ciertamente, el ofendido podría retirarse, no obstante, esto involucraría sacrificar la interacción y su cara. Más allá de la complejidad de estos intercambios, nos interesa resaltar que este análisis en términos de ofensas, ofensores y ofendidos exhibe la naturaleza ritual en la que se funda la interacción.

\footnotetext{
${ }^{21}$ A estas reglas les dedicará un artículo particular (Goffman 1956b).

${ }^{22}$ [Uso el término ritual porque estoy lidiando con actos en los que, a través de un componente simbólico, el actor muestra cuán digno de respeto es o cuán dignos de respeto siente que los otros son. El imaginario de equilibrio es apto aquí porque la profundidad y la intensidad de los esfuerzos correctivos se encuentran bien adaptados a la persistencia e intensidad de las amenazas. La cara de uno, entonces, es una cosa sacra, y el orden expresivo requerido para mantenerla es uno ritual.]
} 
Para concluir este apartado, deseamos destacar el aporte central que presenta este ensayo. Goffman muestra que el orden de la interacción se sustenta en un orden moral que se constituye alrededor del cuidado y la protección de la cara, es decir, en el respeto a los miembros que participan y son reconocidos en este sistema social. Así tenemos que el orden de la interacción puede ser comprendido como un tipo particular y especial de orden moral.

Ciertamente, la interacción no tiene como finalidad u objetivo la protección de la cara, pero su ordenamiento descansa en que esta condición moral esté suficientemente garantizada. Cuando nos encontramos en presencia de otros estamos preocupados por cuidar la cara, principalmente la nuestra, pero también la de los otros. Esta orientación favorece la emergencia y la aceptación de acuerdos de trabajo que, a la vez que protegen y cuidan la cara de los participantes, resultan funcionales para la coordinación de las interacciones. En su búsqueda por salvar la cara, los individuos terminan contribuyendo al orden social. Por esta razón, las reglas de la interacción hallan su fundamento en la necesidad de salvaguardar a las personas que en ella participan. Cuando estas reglas no son cumplidas o corren el riesgo de no serlo, es decir, cuando la cara de alguno de los interlocutores puede ser o es puesta en peligro, la interacción misma es amenazada. Es el carácter ritual de la cara lo que dinamiza un conjunto de estrategias que, buscando proteger o recuperar la cara, resultan efectivas para la continuación del orden de la interacción. ${ }^{23} \mathrm{El}$ acuerdo de trabajo que Goffman coloca en el centro de la respuesta a la pregunta por el orden social en las interacciones, le subyace este complejo moral. El orden de la interacción constriñe a sus participantes a aceptar los acuerdos de trabajo debido a que, a través de ellos, se protege la sacralidad de la cara.

Societies everywhere, if they are to be societies, must mobilize their members as selfregulating participants in social encounters. One way of mobilizing the individual for this purpose is through ritual; he is taught to be perceptive, to have feelings attached to self and self-expressed through face, to have pride, honor, and dignity, to have considerateness, to have tact and a certain amount of poise. There are some of the elements of behavior which must be built into the person if practical use is to be made of him as an interactant, and it is these elements that are referred to in part when one speaks of universal human nature. Universal human nature is not very human thing. By acquiring it, the person becomes a kind of construct, built up not from inner psychic propensities but from moral rules that are impressed upon from without. (Goffman 1967, $44)^{24}$

\footnotetext{
${ }^{23}$ Es interesante notar que en esta formulación se encuentran supuestos los trazos de una teoría general del orden social para la cual el orden no depende de la reproducción de estructuras. Basta con la reproducción del sistema, en este caso de la interacción.

${ }^{24}$ [Las sociedades en todos lados, si ellas deben ser sociedades, deben movilizar sus miembros como participantes auto-regulados en sus encuentros sociales. Una forma de movilizar a los individuos para este propósito es a través del
} 
Algo que quedará pendiente en este análisis es la pregunta de cómo es posible este orden ritual de la interacción. En otros términos, ¿cómo es posible que emerja este orden moral en los encuentros sociales? Cuestión que llevará a Goffman (2004) a la necesidad de definir una teoría de la acción interactiva. En esta dirección, será necesaria una aproximación fenomenológica a la interacción; temática que será objeto de una de sus obras más conocidas, e inmediatamente posterior: La presentación de la persona en la vida cotidiana. ${ }^{25}$

\section{Conclusiones}

A lo largo del trabajo buscamos mostrar las razones que llevaron a Goffman a concebir a la interacción como un campo problemático para la sociología, así como las distintas reflexiones que fueron alumbrando su teoría de la interacción. De esta manera, pudimos observar cómo el interés goffmaniano por los símbolos de status empezó a hacer reconocible el carácter autónomo de la interacción frente a realidades institucionales o macro-estructurales de la sociedad, las cuales encontraban limitaciones para interferir en ellas o controlarlas. A partir de este descubrimiento, empieza a configurarse la inquietud por aquello que regula a las interacciones. De alguna manera, se da a entender que la autonomía de la interacción vuelve insuficientes o inadecuadas a aquellas teorías del orden social cuyo horizonte de análisis lo constituye la sociedad.

Es evidente que Goffman no desestima estas teorías. De hecho, continúa refiriendo a ellas cuando habla de clases sociales, de roles, de posiciones de poder y de normas sociales. El problema que encuentra Goffman en ellas es que dan por supuesto el funcionamiento de las interacciones y su ordenamiento. Si las interacciones reproducen el orden societal es algo que debe ser explicado y no dado por sentado. En este marco, el sociólogo canadiense propone la hipótesis de que son los consensos de trabajo aquellos que viabilizan el orden de la interacción. Es decir, acuerdos pragmáticos que permiten coordinar las acciones en la interacción. La aparente debilidad de esta hipótesis es complementada con el reconocimiento de que el carácter pragmático de estos acuerdos, lejos de ser algo superficial, circunstancial o débil, es, por el contrario, un hecho moral en tanto se

ritual; él es enseñado a ser perceptivo, a tener sentimientos ligados a la persona y a la persona expresada a través de la cara, a tener orgullo, honor, y dignidad, a ser considerado, a tener tacto y cierto monto de equilibrio. Hay algunos elementos del comportamiento que deben ser construidos en la persona si quiere hacer un uso práctico de él como interactuante, y son estos elementos los que son referenciados en parte cuando uno habla de una naturaleza humana universal. La naturaleza humana universal no es una cosa muy humana. Pero adquiriéndola, la persona se vuelve una clase de constructo, construido no desde las propensiones psíquicas interiores sino de las reglas morales que están impresas sobre él desde afuera.]

${ }^{25}$ Podríamos decir que este libro ya no trata estrictamente sobre el orden de la interacción. El eje es la teoría de la acción en situaciones de copresencia. Por esta razón, consideramos que el problema del orden remite a desarrollos previos a la misma, aunque se verá complementado por esta obra.

\section{(c) (1) $(9)$}

Este obra está bajo una licencia de Creative Commons Reconocimiento-NoComercial-SinObraDerivada 4.0 Internacional. 
sustenta en un conjunto de obligaciones y reglas orientadas a la protección de las personas que participan de un encuentro cara a cara.

El valor de la reconstrucción presentada se expresa en dos niveles. Por un lado, han sido usuales las lecturas de Goffman que tienden a retomar sus análisis de la interacción, dejando de lado el conjunto de problemas y de hipótesis que sustentan al mismo. Especialmente, aquellos que tienen que ver con la teoría del orden de la interacción, la cual no suele ser vista como el eje que articula su proyecto intelectual. De hecho, es el fundamento sobre el cual se asienta La presentación de la persona en la vida cotidiana, una de sus obras más difundidas y reconocidas. Sostenemos que resulta difícil comprender esta obra si se desatienden los descubrimientos que el autor hizo previamente alrededor del problema del orden social de la interacción. Quizás en esto radique la razón por la cual diversos lectores de su obra se muestran descontentos con las interpretaciones que se han hecho de ella (Branaman 1997; Jacobsen 2010; Rawls 1987; Maning 2010).

Por otro lado, la recapitulación propuesta da lugar a una reconsideración y potencial revalorización de la teoría goffmaniana en el marco de una teoría de la sociedad y del orden social. Para el caso de la teoría de la sociedad, resulta significativa la distinción interacción/sociedad. Nótese que hablamos de distinción y no de separación. Estamos ante la presencia de dos sistemas diferentes pero que se presuponen. La sociedad se reproduce en las interacciones y estas reproducen la sociedad. La novedad del planteo de Goffman radica en sostener que una teoría de la sociedad no podría obviar la autonomía y la especificidad de la interacción. Del mismo modo, que una teoría de la interacción no podría desarrollarse sin afectar una teoría de la sociedad (siempre que se desee mantener incuestionado el supuesto de que las interacciones ocurren en su interior).

Con respecto a los posibles aportes a la teoría del orden social, la propuesta de Goffman nos hace suponer que esta merece ser revisitada a la luz de las particularidades del orden de la interacción. Orden que parece no requerir ni de consensos profundos, ni de la subordinación a estructuras de poder y/o dominación. En todo caso, estas alternativas son contingentes. De ninguna manera, esto implicaría explicar al orden social a partir de las lógicas interactivas, algo que sería reduccionista y contradictorio con los análisis goffmanianos. Más bien, se trataría de considerar una teoría general que contemple e integre en su desarrollo al orden propio de los encuentros cara a cara.

Este conjunto de implicancias teóricas muestra que Goffman, además de haber sido un observador minucioso de las realidades micro-sociales, fue el teórico que descubrió y desarrolló el problema de la interacción para la sociología. Quizás aún no le hayamos hecho del todo justicia a este descubrimiento.

\section{Referencias}

Alexander, Jeffrey. 2000. "El interaccionismo simbólico: el individualismo y la obra de Blumer y Goffman”. En Las teorías sociológicas desde la segunda guerra mundial. Análisis multidimensional. Barcelona: Gedisa: 176-193.

\section{(c) (1) $\circledast \Theta$}

Este obra está bajo una licencia de Creative Commons Reconocimiento-NoComercial-SinObraDerivada 4.0 Internacional. 
Boltanski, Luc. 1973. "Erving Goffman et le temps du soupçon. A propos de la publication en frangais de la Représentation de soi dans la vie quotidienne". Social Science Information 12 (3): 127-147. https://doi.org/10.1177/053901847301200307

Bourdieu, Pierre. 1983. "Erving Goffman, discoverer of the infinitely small". Theory, Culture and Society 2 (1): 112-113. https://doi.org/10.1177/0263276483002001012

Branaman, Ann. 1997. "Goffman social theory". En The Goffman reader, Lemert y Branaman eds. Massachusetts: Blackwell: 9-41.

Caballero, Juan José. 1998. "La interacción social en Goffman". Revista Española de Investigaciones Sociológicas 83: 121-149. Recuperado de: http://www.reis.cis.es/REIS/PDF/REIS_083_06.pdf

Cadenas, Hugo. 2012. "La desigualdad de la sociedad. Diferenciación y desigualdad en la sociedad moderna". Revista Persona y Sociedad XXVI (2): 51-77. Recuperado de: http://personaysociedad.cl/ojs/index.php/pys/article/view/117/76

Collins, Randall. 1980. "Erving Goffman and the development of modern social theory", en The View from Goffman, Jason Ditton ed. Nueva York: The Macmillan Press: 170-210.

Giddens, Anthony. 1987. "Erving Goffman as a systematic social theorist". En Social Theory and Modern Sociology, Standford: Stanford University Press: 109-139.

Goffman, Erving. 2004. La presentación de la persona en la vida cotidiana, Buenos Aires: Amorrortu.

_1991. "El orden social y la interacción”. En Los momentos y sus hombres, Yves Winkin comp. Barcelona: Paidós: 91-98.

_1983. "The interaction order: American Sociological Association, Presidential address". American Sociological Review 18 (1): 1-17. https://doi.org/10.2307/2095141

_1967. "On face work. An analysis of ritual elements in social interaction”. En Interaction Ritual. Essays on face-to-face behavior. Londres: Pinguin Books: 5-45.

_1956a. "Embarrassment and social organization". American Journal of Sociology. 62 (3): 264 271. Recuperado de: https://www.jstor.org/stable/2772920?seq=1\#page_scan_tab_contents

_1956b. "The nature of deference and demeanor". American Anthropologist, (No. 56): 473-502. https://doi.org/10.1525/aa.1956.58.3.02a00070

_1952. "On cooling the mark out: some aspects of adaptation to failure". Psychiatry 15 (4): 451463. https://doi.org/10.1080/00332747.1952.11022896

_1951. "Symbols of class status". The British Journal of Sociology 2 (4): 294-304. https://doi.org/10.2307/588083

Jacobsen, Michael. 2010. The Contemporary Goffman. New York: Routledge.

Luhmann, Niklas. 1987. "The evolutionary differentiation between society and interaction". En The Micro-Macro Link, Alexander, Giesen, Münch y Smelser eds. Los Angeles: University of California Press: 112-131

\section{(c) (1) $\$($}

Este obra está bajo una licencia de Creative Commons Reconocimiento-NoComercial-SinObraDerivada 4.0 Internacional. 
Manning, Peter. 2010. “Continuities in Goffman. The interaction order”. En The Contemporary Goffman, Michael Jacobsen ed. New York, Routledge: 98-118.

Parsons, Talcott. 1999. El sistema social. Madrid: Alianza Editorial.

Psathas, George. 1980. "Early Goffman and the analysis of face-to-face interaction in Strategic interaction" en The View from Goffman, Jason Ditton ed. Nueva York: The Macmillan Press: 24-51.

Rawls, Anne. 1987. "The interaction order sui generis: Goffman's contribution to social theory". Sociological Theory 5: 136-149. https://doi.org/10.2307/201935

Smith, Greg. 2006. Erving Goffman. New York: Routledge.

Treviño, Javier. 2003. "Introduction: Erving Goffman and the Interaction Order”. En Goffman's Legacy, Javier Treviño ed. Maryland: Rowman and Littlefield.

Winkin, Yves. 1991. "Erving Goffman: retrato del sociólogo joven”. En Los momentos y sus hombres, Yves Winkin ed. Barcelona: Paidós: 13-85. 